\title{
The Peregrine soliton in nonlinear fibre optics
}

\author{
B. Kibler ${ }^{1}$, J. Fatome' ${ }^{1}$, C. Finot ${ }^{1}$, G. Millot ${ }^{1}$, F. Dias ${ }^{2,3}$, G. Genty ${ }^{4}$, N. Akhmediev ${ }^{5}$ and J. M. Dudley ${ }^{6 \star}$
}

The Peregrine soliton is a localized nonlinear structure predicted to exist over $\mathbf{2 5}$ years ago, but not so far experimentally observed in any physical system ${ }^{1}$. It is of fundamental significance because it is localized in both time and space, and because it defines the limit of a wide class of solutions to the nonlinear Schrödinger equation (NLSE). Here, we use an analytic description of NLSE breather propagation ${ }^{2}$ to implement experiments in optical fibre generating femtosecond pulses with strong temporal and spatial localization, and near-ideal temporal Peregrine soliton characteristics. In showing that Peregrine soliton characteristics appear with initial conditions that do not correspond to the mathematical ideal, our results may impact widely on studies of hydrodynamic wave instabilities where the Peregrine soliton is considered a freak-wave prototype ${ }^{3-7}$.

Solitons are localized waves arising from nonlinear and dispersive interactions, and are central objects of nonlinear science. The well-known envelope solitons of the NLSE have been studied in many different systems including plasmas, optical fibres and cold atoms ${ }^{8-10}$. In addition to envelope solitons, the NLSE admits other classes of localized structure, and there has been significant interest in spatio-temporal breather solutions that undergo periodic energy exchange with a finite background ${ }^{11,12}$. However, despite extensive mathematical studies $^{4,5}$, experiments have been limited to only a small number of discrete systems ${ }^{9,13}$. Indeed, to our knowledge no studies have explicitly characterized nonlinear breather localization in any system described by the continuous NLSE. As a result, predictions such as Peregrine's that are central to nonlinear wave theory have remained untested.

In a sense, this is surprising because the theory of NLSE breather evolution also describes induced modulation instability, a process extensively studied in hydrodynamics and fibre optics ${ }^{14-18}$. Experiments in optics, however, have been strongly motivated by telecommunications goals to generate high-contrast pedestalfree pulses ${ }^{19-22}$, and the opportunity to characterize solitons on a finite background seems to have been overlooked. Indeed, even fundamental studies of Fermi-Pasta-Ulam recurrence in modulation instability have been carried out using initial conditions far from those that would excite Peregrine soliton features ${ }^{23}$. Here, we report experiments in optical fibre specifically designed to study breather evolution in a regime approaching the excitation of the Peregrine soliton. We demonstrate explicitly its spatio-temporal localization and, at the point of maximum temporal compression, use frequency-resolved optical gating (FROG) to explicitly measure the temporal soliton characteristics on a finite background. Our results are in very good agreement with numerical simulations and Peregrine's analytic prediction.
Our experiments are designed using the breather formalism of ref. 2. With dimensionless field $\psi(\xi, \tau)$, the self-focusing NLSE is:

$$
i \frac{\partial \psi}{\partial \xi}+\frac{1}{2} \frac{\partial^{2} \psi}{\partial \tau^{2}}+|\psi|^{2} \psi=0
$$

Here $\xi$ and $\tau$ are normalized distance and time, and induced modulation instability involves the evolution along $\xi$ of a temporally modulated continuous wave towards a train of ultrashort compressed pulses followed by a return phase of broadening towards the initial state. Although the general evolution can be complex, it has recently been shown ${ }^{24}$ that the compression dynamics can be described for a wide range of initial conditions by the analytic Akhmediev breather ${ }^{2,4}$ :

$$
\psi(\xi, \tau)=\left[\frac{(1-4 a) \cosh (b \xi)+\sqrt{2 a} \cos (\Omega \tau)+i b \sinh (b \xi)}{\sqrt{2 a} \cos (\Omega \tau)-\cosh (b \xi)}\right] \mathrm{e}^{i \xi}
$$

Here $\Omega$ is the dimensionless modulation frequency, $a=1 / 2(1-$ $\Omega^{2} / 4$ ), where $0<a<1 / 2$ determines the frequencies that experience gain and $b=[8 a(1-2 a)]^{1 / 2}$ determines the instability growth. Dimensional transformations are given below, and dimensional forms of both equations (1) and (2) are given in the Methods section.

Figure 1 shows how the breather characteristics depend strongly on modulation frequency. As the modulation parameter $a$ increases, the temporal separation between adjacent peaks increases at the same time as the compressed temporal width of each individual peak decreases. This leads to a greater temporal localization (defined below) as $a$ approaches the limiting value of $1 / 2$. The evolution at $a=1 / 4$ is associated with maximum modulation-instability gain, and is indeed the regime of previous experiments ${ }^{19,23}$. The limiting solution for $a \rightarrow 1 / 2$ derived by Peregrine has a particular fractional form that has led this class of solution to be described as a 'rational soliton'

$$
\psi(\xi, \tau)=\left[1-\frac{4(1+2 i \xi)}{1+4 \tau^{2}+4 \xi^{2}}\right] \mathrm{e}^{i \xi}
$$

The figure also plots the ideal Peregrine soliton to show the breather evolution approaching this solution as the overlap between adjacent peaks reduces with increasing $a$.

Figure 2a shows in more detail the dependence on modulation parameter of the breather profile at the point of maximum temporal compression $\xi=0$. The pseudocolour plot of $|\psi(0, \tau)|^{2}$ highlights the increased peak localization as $a \rightarrow 1 / 2$, with the right panels illustrating how the intensity of the half-period breather peak

\footnotetext{
${ }^{1}$ Laboratoire Interdisciplinaire Carnot de Bourgogne, UMR 5209 CNRS - Université de Bourgogne, Dijon, France, ${ }^{2}$ Centre de Mathématiques et de Leurs Applications (CMLA), ENS Cachan, 94230 Cachan, France, ${ }^{3}$ UCD School of Mathematical Sciences, University College Dublin, Belfield, Dublin 4, Ireland, ${ }^{4}$ Tampere University of Technology, Optics Laboratory, FI-33101 Tampere, Finland, ${ }^{5}$ Optical Sciences Group, Research School of Physics and Engineering, Institute of Advanced Studies, The Australian National University, Canberra ACT 0200, Australia, ${ }^{6}$ Institut FEMTO-ST, UMR 6174 CNRS-Université de Franche-Comté, 25030 Besançon, France. *e-mail: john.dudley@univ-fcomte.fr.
} 

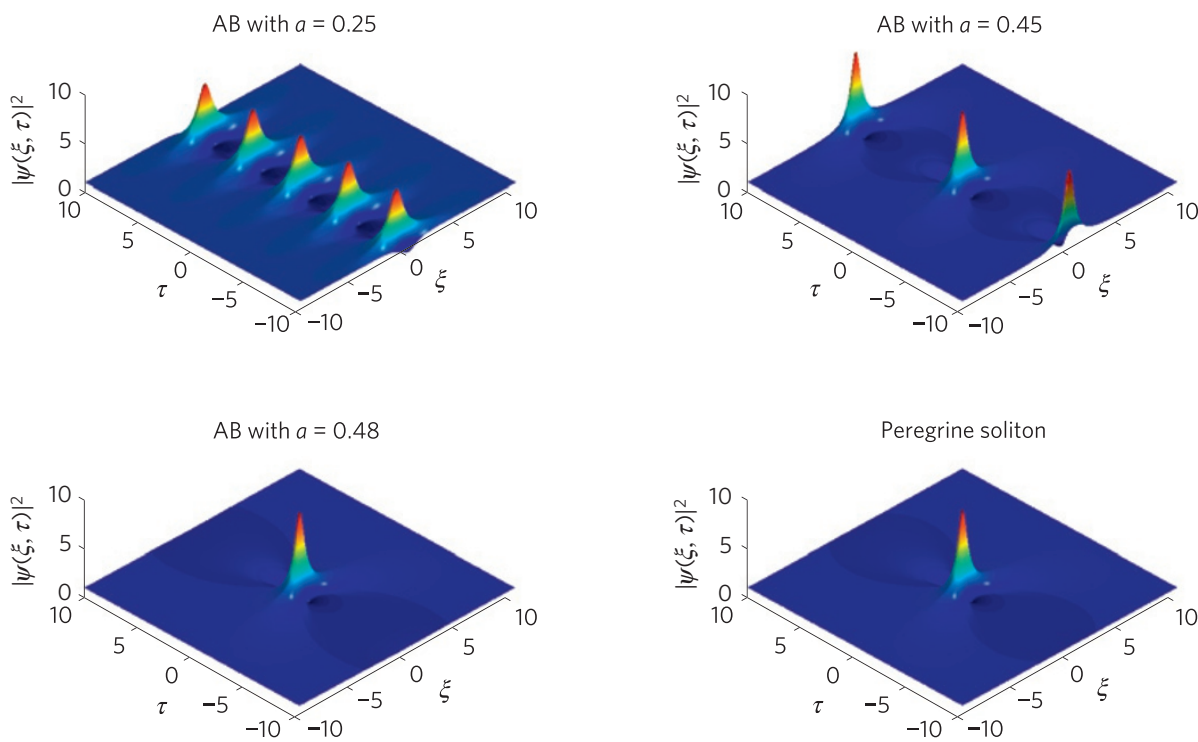

Figure 1 Plotted Akhmediev breather solutions using equation (2) for modulation parameter $a=0.25, a=0.45$ and $a=0.48$, as well as the ideal Peregrine soliton of equation (3), the limiting case of the Akhmediev breather as $a \rightarrow \mathbf{1 / 2}$. Maximum temporal compression occurs at normalized distance $\xi=0$. The differences between the Akhmediev breather $(\mathrm{AB})$ with $a=0.48$ and the Peregrine soliton can be seen with close inspection of the decay of the peak to the wings; they are shown more clearly in Fig. 2.

(shaded region delimited by arrows) approaches the Peregrine soliton (solid line) for $a>0.4$. These results are important in showing that this parameter regime yields characteristic Peregrine soliton features in the temporal envelope even though the ideal solution exists only asymptotically in the limit of zero modulationinstability gain $(b \rightarrow 0$ as $a \rightarrow 1 / 2)$.

The emergence of Peregrine soliton characteristics with increased temporal localization is also associated with increasing spatial localization. This is because the modulation-instability recurrence period also increases asymptotically as $a \rightarrow 1 / 2$. To discuss this quantitatively, we introduce localization measures in terms of ratios of the temporal and spatial periods relative to the individual temporal and spatial peak half-widths. These can be readily calculated or determined from numerical simulations of the NLSE as a function of modulation parameter (see the Methods section). The solid lines in Fig. $2 b$ plot (i) temporal localization $\tau_{\mathrm{per}} / \delta \tau_{o}$, (ii) spatial localization $\xi_{\text {per }} / \delta \xi_{o}$ and (iii) their product $\left(\tau_{\text {per }} / \delta \tau_{o}\right)\left(\xi_{\text {per }} / \delta \xi_{o}\right)$, which defines spatio-temporal localization. The regime of rapidly increasing 'strong localization' for $a>0.4$ is where the right panels in Fig. $2 \mathrm{a}$ show Peregrine soliton characteristics in the temporal envelope. As discussed below, this regime is accessible in our experiments. This analysis is thus highly significant because it shows how Peregrine soliton characteristics can appear experimentally, even though the ideal mathematical asymptotic limit can never be reached in practice.

Figure 3 shows our experimental set-up (see the Methods section). An input field $A\left(z=z_{0}, T\right)=\sqrt{P_{0}}\left[1+\alpha_{\bmod } \exp \left(i \omega_{\bmod } T\right)\right]$ is injected into fibre with group velocity dispersion $\beta_{2}\left(\mathrm{~s}^{2} \mathrm{~m}^{-1}\right)$ and nonlinearity $\gamma\left(\mathrm{W}^{-1} \mathrm{~m}^{-1}\right)$. The input power is $P_{0}$, and $\alpha_{\bmod }$ and $\omega_{\text {mod }}$ are modulation strength and frequency. The dimensional field $A(z, T)\left(\mathrm{W}^{1 / 2}\right)$ is $A=P_{0}{ }^{1 / 2} \psi$. Defining a characteristic length $L_{\mathrm{NL}}=\left(\gamma P_{0}\right)^{-1}$ and timescale $T_{0}=\left(\left|\beta_{2}\right| L_{\mathrm{NL}}\right)^{1 / 2}$, dimensional distance $z(\mathrm{~m})$ and time $T(\mathrm{~s})$ are related to the normalized parameters by $z=\xi L_{\mathrm{NL}}$ and $T=\tau T_{0}$. The frequency $\omega_{\bmod }$ is related to the modulation parameter $a$ by $2 a=\left[1-\left(\omega_{\bmod } / \omega_{\mathrm{c}}\right)^{2}\right]$ with $\omega^{2}{ }_{c}=4 \gamma P_{0} /\left|\beta_{2}\right|$. Modulation-instability gain is seen for modulation parameters $0<a<1 / 2$, which corresponds to $\omega_{\mathrm{c}}>\omega_{\text {mod }}>0$. With these definitions, evolution towards the Peregrine soliton as $a \rightarrow 1 / 2$ corresponds to the limit where $\omega_{\text {mod }} \rightarrow 0$, accessible in practice by beating two narrow-linewidth lasers to create an initial low-frequency-modulated wave.
We used an input field obtained from a pump laser at $\lambda_{\mathrm{p}}=1,554.53 \mathrm{~nm}$ mixed with a closely spaced tunable signal at $\lambda_{\mathrm{s}}$, with $\alpha_{\text {mod }}=0.225$ (see the Methods section for other parameters). Experiments first studied two-dimensional localization dynamics as a function of modulation parameter $a$. Although we use a fibre of fixed length, the spatial dynamics in $\xi$ were readily measured by varying pump power (recall $\xi=z \gamma P_{0}$ ). By changing the pumpsignal detuning to vary $a$ while studying dynamical evolution by varying $P_{0}$, the temporal and spatial localization parameters defined above were determined from the $\xi$ and $\tau$ dependence of the measured autocorrelation function $g(\xi, \tau)=\left\langle|\psi(\xi, t)|^{2}|\psi(\xi, t-\tau)|^{2}\right\rangle$. Figure $2 \mathrm{~b}$ shows that the measured localization parameters are in very good agreement with predictions. This verifies that we can enter experimentally the divergent regime where Peregrine soliton characteristics are observed with the values of $a>0.4$ accessible with our set-up.

Figure 4 shows detailed measurements of dynamics and the compressed temporal profile for strong localization with $a=0.42$. Figure $4 \mathrm{a}$ shows simulations plotting the expected evolution of $|\psi(\xi, \tau)|^{2}$ over the range of $\xi$ varied in our experiments (see the Methods section). Figure $4 \mathrm{~b}, \mathrm{c}$ shows the simulated autocorrelation function and spectrum. These results are compared to experiment (with no free parameters), exhibiting very good agreement. The autocorrelation results in Fig. 4b (i) are particularly clear in showing the two-dimensional localization at the distance of maximum compression when $\xi=2.5$. To illustrate the growth-decay intensity dynamics explicitly, Fig. 4b (ii) shows an increase in autocorrelation peak signal with $\xi$. Here, experiments (points) are compared with simulations (line).

Detailed temporal measurements using FROG were carried out at $\xi=2.5$ where Peregrine soliton characteristics are expected at maximum compression. Figure $5 \mathrm{a}, \mathrm{b}$ shows the measured and retrieved FROG traces. The retrieved intensity and phase (see the Methods section) are shown as the blue line with markers in Fig. $5 \mathrm{~d}$. The peak power of the retrieved profile is calculated from the measured output power with no free parameters. Experimental results are compared to numerical simulations (red line), and we see excellent agreement. Figure $5 c$ shows the FROG trace of the simulated field. The grey line plots the analytic Peregrine soliton from equation (3), with maximum peak power from theory of 


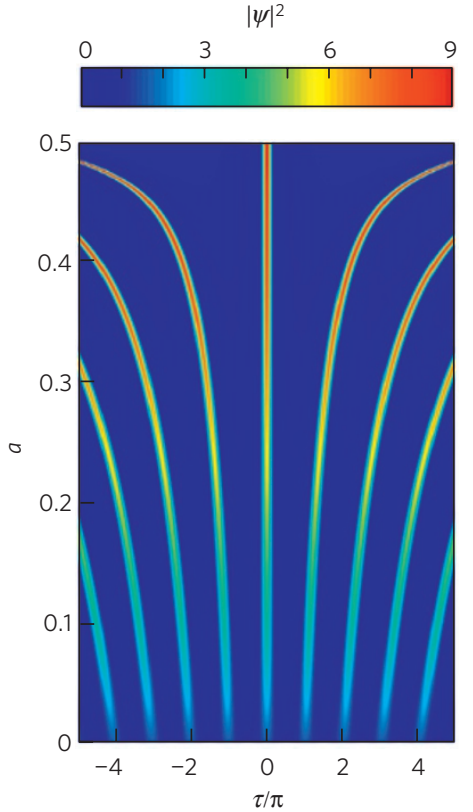

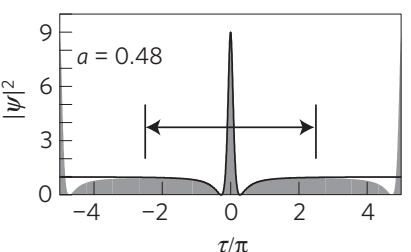
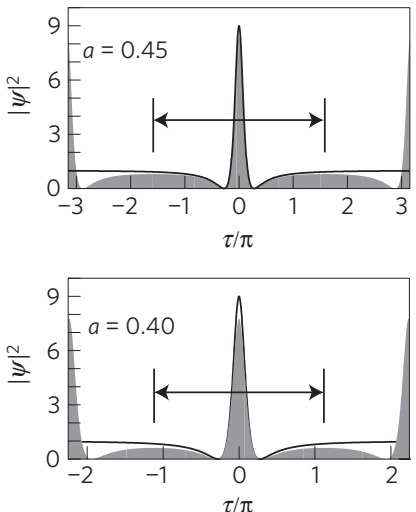

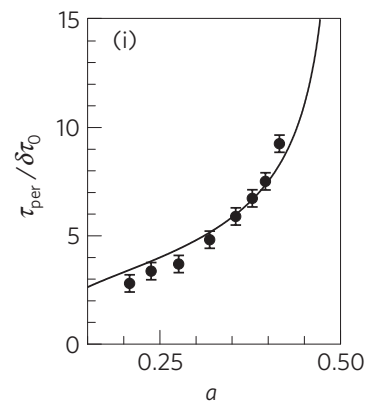

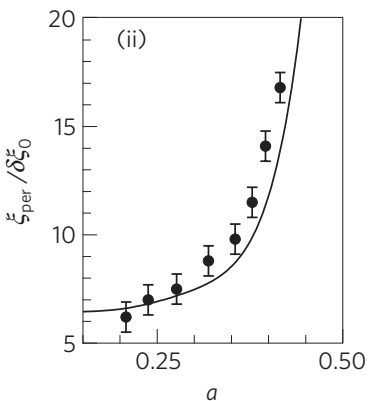

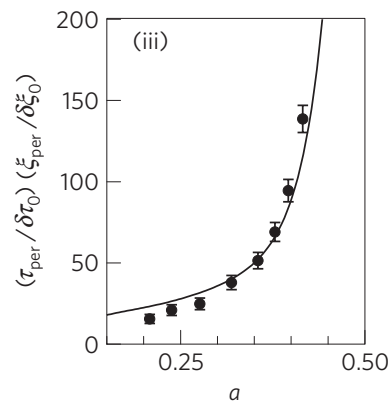

Figure 2 | Evolution towards Peregrine soliton characteristics with increasing modulation parameter for temporal profile characteristics and localization behaviour. a, Numerical results using equation (2) showing the temporal characteristics of the maximally compressed breather. The pseudocolour plot shows the maximally compressed breather intensity $|\psi(0, t)|^{2}$ as the modulation parameter varies over $0<a<0.5$. Limiting cases $a=0$ and $a=0.5$ correspond to a plane wave and the Peregrine soliton, respectively. The panels on the right illustrate how for $a>0.4$, the half-period breather profile (shaded region delimited by arrows) approaches the ideal Peregrine soliton envelope (solid line). The timebase is normalized relative to the minimum modulation period of $\tau=\pi$ as $a \rightarrow 0$. Note the expanding timebase as the modulation parameter increases. $\mathbf{b}$, The dependence on modulation parameter of (i) temporal, (ii) spatial and (iii) spatio-temporal localization parameters as defined in the text. The figure highlights the divergent nature of localization as $a$ increases above 0.4. The solid lines represent analytical/numerical results (see the Methods section) and the points with error bars are obtained from experiment. The error bars in $\mathbf{b}$ are calculated from the average over 5 series of repeated measurements of temporal and spatial localization at each value of $a$, taking into account estimated errors of $\pm 5 \%$ in measurements of temporal width and power.
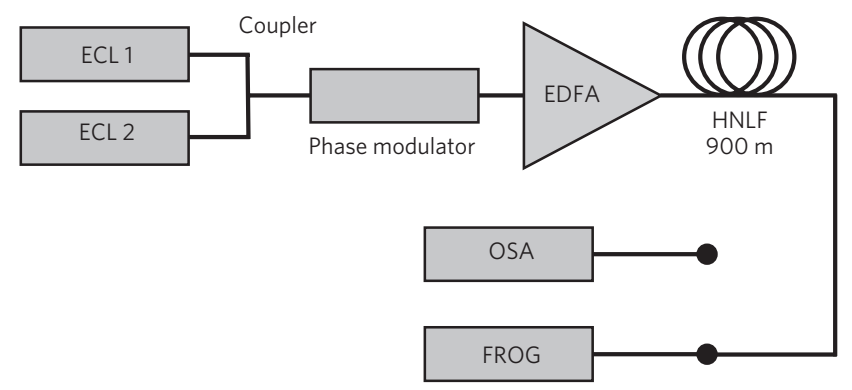

\section{Autocorrelator}

Figure 3 | Experimental set-up. ECL: external-cavity laser; OSA: optical spectrum analyser; FROG: frequency-resolved optical gating. HNLF: highly nonlinear fibre. EDFA: erbium-doped fibre amplifier.
$9 P_{0}=2.7 \mathrm{~W}$. On the right axis, the intensity for all curves is normalized to that of the dimensionless $|\psi(0, \tau)|^{2}$.

These measurements confirm the expected temporal features of the Peregrine soliton-a temporally localized peak ( $400 \mathrm{fs}$ duration) surrounded by a non-zero background. The FROG measurements also confirm the different signs of the peak and background amplitudes through the measured relative $\pi$ phase difference. The measured phase profile (blue line and markers) in the vicinity of the intensity null (indicated by the arrow) is shown in Fig. 5d and compared to that expected for the Peregrine soliton (grey line). Finally, in Fig. 5e we plot the measured spectral intensities (blue) compared to simulation (red markers), and the analytic spectrum $|\tilde{\psi}|^{2} \sim \exp \left[-2 \pi|\nu|\left(\left|\beta_{2}\right| / \gamma P_{0}\right)^{1 / 2}\right]$ for the ideal Peregrine soliton (grey). Note that this spectrum is calculated for the time-varying envelope component so that the delta-function component at the pump is not shown, but the analytic spectrum reproduces very well the decay of the measured sideband amplitudes. 
a

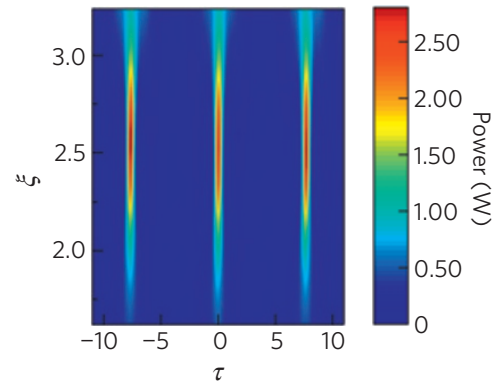

b

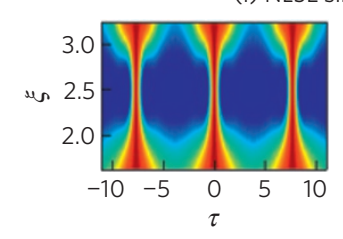

(i) NLSE simulation and experiment

$$
\text { C }
$$

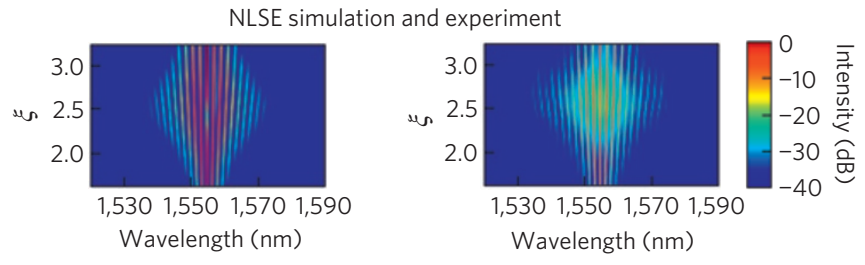

(ii) Peak evolution
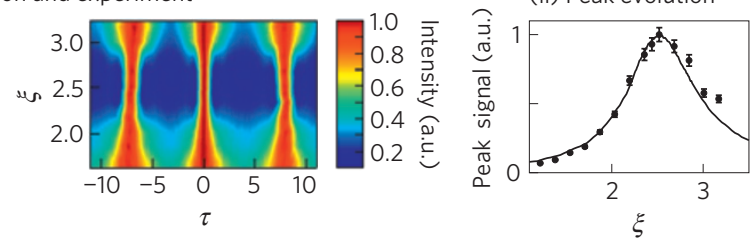

Figure 4 | The variation in temporal and spectral characteristics with normalized propagation distance to confirm expected dynamical evolution. a, NLSE simulations of temporal intensity evolution. b. Autocorrelation dynamics showing: (i) temporal compression and broadening for simulated (left) and experimental (right) autocorrelation traces; (ii) peak autocorrelation signal for simulated (solid line) and experiments (points with error bars). The

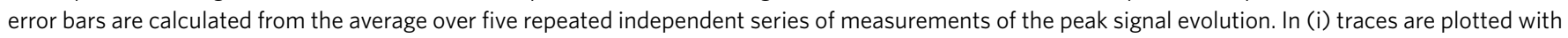
normalization to a peak of unity at each $\xi$ to highlight temporal localization; in (ii) normalization is with respect to the global peak signal intensity with propagation to highlight spatial localization. c, Comparing simulated (left) and experimental (right) spectral dynamics.

a

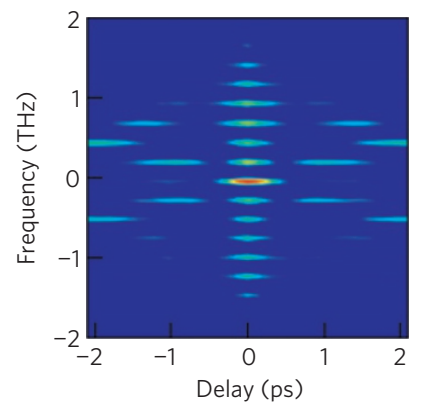

b

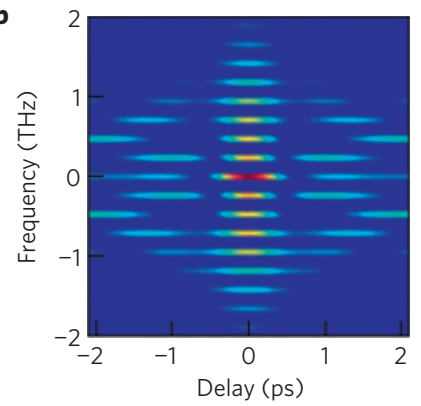

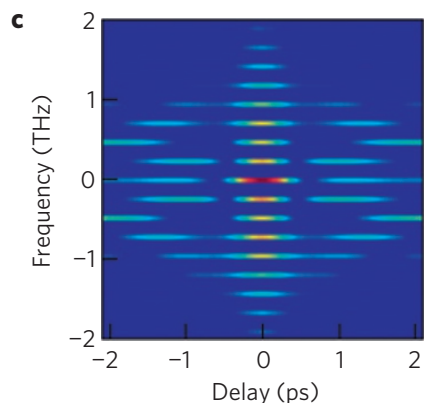

d

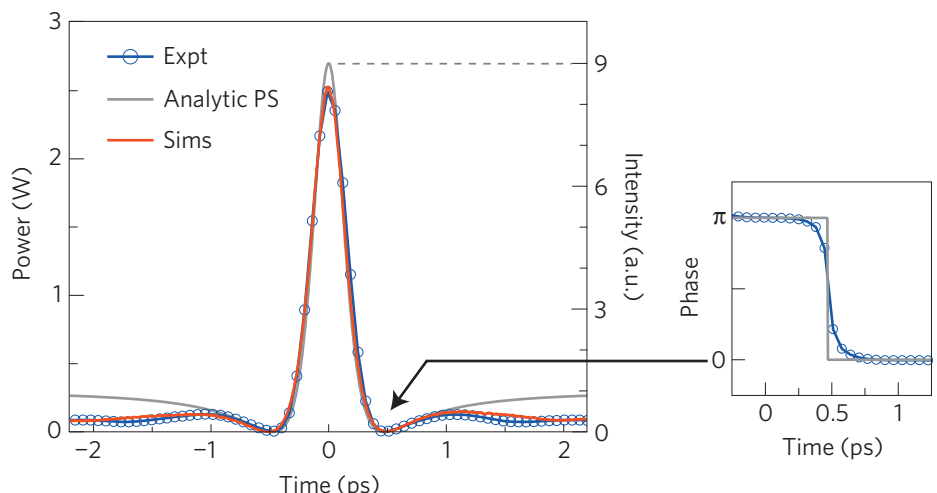

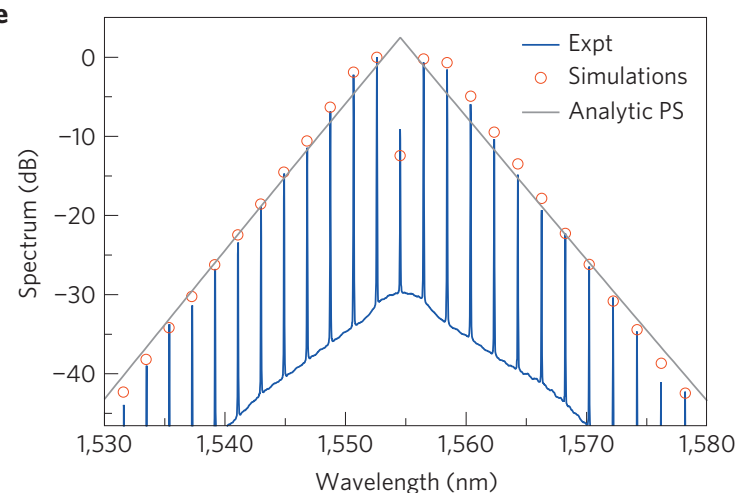

Figure 5 | Experimental results showing the measured temporal characteristics of the maximally compressed pulse at $\xi=2.5$, and comparison with the predicted Peregrine soliton. a-c, Measured (a), retrieved (b) and simulated (c) FROG traces. d, Intensity and phase from experiment (blue), simulation (red) and for the ideal Peregrine soliton (grey). e, Corresponding spectral characteristics from experiment (blue), simulation (red markers, shown at peaks only for clarity) and for the ideal Peregrine soliton (grey). The Peregrine soliton spectrum is of the time-varying envelope component so that the delta-function component at the pump wavelength is not shown.

These experiments represent the first amplitude and phase measurements of a nonlinear breather structure in any continuous NLSE soliton-supporting system. The results show the existence of a strongly localized temporal peak on a non-zero background, and confirm Peregrine's theoretical predictions of a rational soliton envelope. Our results highlight how experiments in optics using readily available components and advanced pulse metrology can be used to conveniently test more general theories of nonlinear waves ${ }^{25}$. We anticipate applications in establishing links between optical and hydrodynamic extreme events ${ }^{26}$ and 
in studying modulation-instability dynamics in other systems ${ }^{27}$. In showing that Peregrine soliton characteristic are excited even for non-ideal perturbation frequencies, our results may impact on the search for oceanic rogue-wave forecasting signatures in meteorological data.

\section{Methods}

For completeness, we first give dimensional forms of the NLSE and breather solutions equations (1) and (2) respectively. The dimensional NLSE is given by:

$$
i \frac{\partial A}{\partial z}-\frac{\beta_{2}}{2} \frac{\partial^{2} A}{\partial T^{2}}+\gamma|A|^{2} A=0
$$

with $\beta_{2}<0$ for the self-focusing form (that is, fibre anomalous dispersion). The breather evolution in $z$ and $T$ is given in dimensional form as:

$$
A(z, T)=\sqrt{P_{0}} \frac{(1-4 a) \cosh \left(b z / L_{\mathrm{NL}}\right)+i b \sinh \left(b z / L_{\mathrm{NL}}\right)+\sqrt{2 a} \cos \left(\omega_{\bmod } T\right)}{\sqrt{2 a} \cos \left(\omega_{\bmod } T\right)-\cosh \left(b z / L_{\mathrm{NL}}\right)}
$$

The numerical results in Figs 1 and 2 were obtained directly from equation (2) In numerical simulations solving the NLSE equation (1), we used a standard split-step scheme ${ }^{28}$

The localization properties shown in Fig. 2 are defined in terms of normalized variables as follows. A measure of time-domain localization can be derived analytically from the form of profile of the breather solution at the point of maximum temporal compression: $|\psi(0, \tau)|^{2}=([(1-4 a)+\sqrt{2 a} \cos (\Omega \tau)] /[\sqrt{2 a} \cos (\Omega \tau)-1])^{2}$. We define the profile temporal width $\delta \tau_{0}$ as the position when the intensity is zero-valued adjacent to the peak. Zeros appear in the profile for $a>1 / 8$, and the ratio between the period $\tau_{\text {per }}=2 \pi / \Omega$ and the temporal width can then be expressed analytically as: $\tau_{\text {per }} / \delta \tau_{0}=2 \pi \cos ^{-1}[(4 a-1) / \sqrt{2 a}]$. This expression is that used to plot the solid line in Fig. $2 \mathrm{~b}$ (i). Under non-ideal initial conditions, multiple spatial recurrence periods of breather evolution are observed, and longitudinal localization is not amenable to straightforward analysis. We therefore used numerical simulations of the NLSE plotting peak intensity evolution with $\xi$. This allows us to determine the spatial period $\xi_{\text {per }}$ and the half-width $\delta \xi_{\mathrm{o}}$ of the intensity evolution with $\xi$ to yield the ratio $\xi_{\text {per }} / \delta \xi_{0}$. The solid line in Fig. 2b (ii) shows a smooth polynomial fit to simulations that we carried out for varying $a$. At the parameters used in our experiments for the detailed temporal analysis $(a=0.42)$, the measured spatial localization factor exceeded 10

In our experiments, the initial signals (pump and seed) were generated from two telecommunications-grade external-cavity lasers (ECL-OSICS model $1560-\mathrm{PM}$ ) with intrinsic linewidths $<200 \mathrm{kHz}$. The fibre used was $900 \mathrm{~m}$ of highly nonlinear fibre (OFS Specialty Fiber) with $\beta_{2}=-8.85 \times 10^{-28} \mathrm{~s}^{2} \mathrm{~m}^{-1}$ and $\gamma=0.01 \mathrm{~W}^{-1} \mathrm{~m}^{-1}$ at $\lambda_{\mathrm{p}}$. The fibre was dispersion-flattened to have low third-order dispersion $\beta_{3}=1.331 \times 10^{-41} \mathrm{~s}^{3} \mathrm{~m}^{-1}$ around $1,550 \mathrm{~nm}$. Fibre loss was $1 \mathrm{~dB} \mathrm{~km}^{-1}$. A phase modulator was used to broaden the narrow intrinsic external-cavity-laser linewidths to $\sim 100 \mathrm{MHz}$ so as to suppress Brillouin scattering in the fibre at the power levels used in our experiments ${ }^{28}$. Both the pump and the seed were then amplified to the power levels used in the experiments by means of an erbium-doped fibre amplifier (EDFA-IPG model EAD-1-C-PM). Note that the injection set-up was all-polarization maintaining to maximize the modulation-instability process occurring in the optical fibre. A low-noise amplifier was used so as to clearly favour the induced breather dynamics over spontaneous broadband modulation instability. Indeed, the limiting factor in reducing the modulation frequency between the pump and the signal so as to approach the ideal case of $a \rightarrow 1 / 2$ is the decreasing gain for the stimulated process relative to the spontaneous growth of sideband content, which occurs over a continuous range from the pump to the maximum frequency $\omega_{\mathrm{c}}$. In our experiments this limited the maximum attainable value of $a=0.42$

For the experimental results in Fig. 4, we varied $P_{0}$ from 0.2 to $0.4 \mathrm{~W}$ while varying $\omega_{\bmod }$ over $196.7-278.2 \mathrm{GHz}$ (by tuning $\lambda_{\mathrm{s}}$ from 1,556.12-1,556.77 nm) to maintain constant $a=0.42$. Note that the spectral measurements in Fig. 4 are plotted against wavelength to show the change in modulation frequency that ensures that $a$ is held constant. The point of maximum temporal compression at $\xi=2.5$ corresponded to $P_{0}=0.30 \mathrm{~W}$ and $\omega_{\bmod }=241 \mathrm{GHz}$. For the results in Fig. 5, the FROG technique used a second-harmonic generation implementation, and retrieval of the intensity and phase of the underlying field from the measured FROG trace was carried out using a generalized projections algorithm, adapted for finite background fields and/or periodic pulse trains ${ }^{29}$. We measured five periods of the FROG trace although for clarity only the central period is shown in Fig. 5. The non-collinear autocorrelation measurements were carried out using an independent second-harmonic generation autocorrelator providing an important verification of measurement fidelity. Both autocorrelation and FROG measurements are self-referencing and thus insensitive to relative source phase variation. Retrieval was carried out using a $256 \times 256$ grid and the FROG error for the result in Fig. $5 \mathrm{~d}$ was $G=0.005$, a typical error for a periodic FROG trace ${ }^{29}$ Optical spectra were measured using a 0.02 -nm-resolution bandwidth optical spectrum analyser (Yokogawa-AQ6370). In practice, optimization of the injected power to obtain maximum compression for the available fibre length of $900 \mathrm{~m}$ was used by continuous monitoring of spectral and autocorrelation measurements for various input powers around $0.3 \mathrm{~W}$.

The NLSE simulations shown in Fig. 4 for our experimental conditions considered an input corresponding to a weakly modulated continuous-wave field with modulation frequency and depth corresponding to experiment. A low level of bandwidth-limited noise at $-50 \mathrm{~dB}$ modelled the effect of amplified spontaneous emission in the EDFA and a phenomenological one photon per mode background was also included to model quantum noise ${ }^{30}$. The effect of fibre loss was included through a correction to the propagation distance (by using the effective length defined as $L_{\text {eff }}=[1-\exp (-\alpha L)] / \alpha$, where $\alpha$ accounts for fibre losses $\left.{ }^{28}\right)$ when plotting the experimental results. This approach is satisfactory in allowing the simulations to be used to confirm experimental observation of temporal compression and localization in the breather evolution. On the other hand, when comparing the retrieved intensity and phase of the Peregrine soliton, the simulations in Fig. 5 used generalized NLSE simulations including fibre third-order dispersion and spontaneous Raman scattering ${ }^{30}$. However, we found that the generalized NLSE simulations yield essentially the same temporal and spectral characteristics as a NLSE model at the power levels considered.

\section{Received 27 February 2010; accepted 29 June 2010;} published online 22 August 2010

\section{References}

1. Peregrine, D. H. Water waves, nonlinear Schrödinger equations and their solutions. J. Aust. Math. Soc. Ser. B 25, 16-43 (1983).

2. Akhmediev, N. \& Korneev, V. I. Modulation instability and periodic solutions of the nonlinear Schrödinger equation. Theor. Math. Phys. 69, 1089-1093 (1986).

3. Henderson, K. L, Peregrine, D. H. \& Dold, J. W. Unsteady water wave modulations: Fully nonlinear solutions and comparison with the nonlinear Schrödinger equation. Wave Motion 29, 341-361 (1999).

4. Dysthe, K. B. \& Trulsen, K. Note on breather type solutions of the NLS as models for freak-waves. Phys. Scripta 82, 48-52 (1999).

5. Kharif, C., Pelinovsky, E. \& Slunyaev, A. Rogue Waves in the Ocean (Springer-Verlag, 2009).

6. Akhmediev, N., Soto-Crespo, J. M. \& Ankiewicz, A. Extreme waves that appear from nowhere: On the nature of rogue waves. Phys. Lett. A 373, 2137-2145 (2009).

7. Shrira, V. I. \& Geoigjaev, V. V. What makes the Peregrine soliton so special as a prototype of freak waves? J. Eng. Math. 67, 11-22 (2010).

8. Taylor, J. R. (ed.) Optical Solitons Theory \& Experiment (Cambridge Univ. Press, 1992).

9. Dauxois, Th. \& Peyrard, M. Physics of Solitons (Cambridge Univ. Press, 2006).

10. Denschlag, J. et al. Generating solitons by phase engineering of a Bose-Einstein condensate. Science 287, 97-101 (2000).

11. Fermi, E., Pasta, J. \& Ulam, S. in Collected Papers of Enrico Fermi (ed. Segre, E.) 978-988 (Univ. Chicago Press, 1965).

12. Akhmediev, N. \& Ankiewicz, A. Solitons, Nonlinear Pulses and Beams (Chapman and Hall, 1997).

13. Sato, M. \& Sievers, A. J. Direct observation of the discrete character of intrinsic localized modes in an antiferromagnet. Nature 432, 486-488 (2004).

14. Bespalov, V. I. \& Talanov, V. J. Filamentary structure of light beams in nonlinear liquids. JETP Lett. 3, 307-310 (1966).

15. Benjamin, T. B. \& Feir, J. E. The disintegration of wavetrains on deep water. Part 1: Theory. J. Fluid Mech. 27, 417-430 (1967).

16. Hasegawa, A. Generation of a train of soliton pulses by induced modulational instability in optical fibres. Opt. Lett. 9, 288-290 (1984).

17. Tai, K., Tomita, A., Jewell, J. L. \& Hasegawa, A. Generation of subpicosecon solitonlike optical pulses at $0.3 \mathrm{THz}$ repetition rate by induced modulational instability. Appl. Phys. Lett. 49, 236-238 (1986).

18. Hart, D. L., Judy, A. F., Roy, R. \& Beletic, J. W. Dynamical evolution of multiple four-wave-mixing processes in an optical fiber. Phys. Rev. E 57, 4757-4774 (1998)

19. Greer, E. J., Patrick, D. M., Wigley, P. G. J. \& Taylor, J. R. Generation of $2 \mathrm{THz}$ repetition rate pulse trains through induced modulational instability. Electron. Lett. 25, 1246-1248 (1989).

20. Mamyshev, P. V., Chernikov, S. V., Dianov, E. M. \& Prokhorov, A. M. Generation of a high-repetition-rate train of practically noninteracting solitons by using the induced modulational instability and Raman self-scattering effects. Opt. Lett. 15, 1365-1367 (1990).

21. Trillo, S. \& Wabnitz, S. Dynamics of the nonlinear modulational instability in optical fibres. Opt. Lett. 16, 986-988 (1991).

22. Fatome, J., Pitois, S. \& Millot, G. 20-GHz-to-1-THz repetition rate pulse sources based on multiple four-wave mixing in optical fibers. IEEE J. Quantum Electron. 42, 1038-1046 (2006). 
23. Van Simaeys, G., Emplit, Ph. \& Haelterman, M. Experimental demonstration of the Fermi-Pasta-Ulam recurrence in a modulationally unstable optical wave. Phys. Rev. Lett. 87, 033902 (2001).

24. Dudley, J. M., Genty, G., Dias, F., Kibler, B. \& Akhmediev, N. Modulation instability, Akhmediev Breathers and continuous wave supercontinuum generation. Opt. Exp. 17, 21497-21508 (2009).

25. Dudley, J. M., Finot, C., Richardson, D. J. \& Millot, G. Self similarity in ultrafast nonlinear optics. Nature Phys. 3, 597-603 (2007).

26. Solli, D. R., Ropers, C., Koonath, P. \& Jalali, B. Optical rogue waves. Nature 450, 1054-1057 (2007).

27. Doktorov, E. V., Rothos, V. M. \& Kivshar, Y. S. Full-time dynamics of modulational instability in spinor Bose-Einstein condensates. Phys. Rev. A 76, 013626 (2007).

28. Agrawal, G. P. Nonlinear Fibre Optics 4th edn (Academic, 2007).

29. Dudley, J. M. et al. Complete intensity and phase characterisation of optical pulse trains at THz repetition rates. Electron. Lett. 35, 2042-2044 (1999).

30. Dudley, J. M., Genty, G. \& Coen, S. Supercontinuum generation in photonic crystal fibre. Rev. Mod. Phys. 78, 1135-1184 (2006).

\section{Acknowledgements}

We acknowledge support from the French Agence Nationale de la Recherche projects MANUREVA ANR-08-SYSC-019 and IMFINI ANR-09-BLAN-0065, the Academy of Finland Research grants 132279 and 130099, the 2008 Framework Program for Research, Technological development and Innovation of the Cyprus Research Promotion Foundation under the Project ASTI/0308(BE)/05 and the Australian Research Council Discovery Project scheme DP0985394.

\section{Author contributions}

B.K., J.F., C.F. and J.M.D. carried out experiments. The development of analytical tools and simulations was carried out by B.K., G.M., G.G., F.D., N.A. and J.M.D. All authors participated in the analysis and interpretation of the results and the writing of the paper.

\section{Additional information}

The authors declare no competing financial interests. Reprints and permissions information is available online at http://npg.nature.com/reprintsandpermissions. Correspondence and requests for materials should be addressed to J.M.D. 